\title{
Minimally Invasive Esophagectomy: Are We There Yet?
}

\author{
Zane Hammoud, MD \\ Department of Surgery, Ascension Providence Hospital, Southfield, MI
}

The first minimally invasive esophagectomy (MIE) was performed in the early 1990s, and since that time, numerous reports have demonstrated the feasibility and safety of the procedure. In addition, numerous reports have documented excellent short- and long-term outcomes of MIE. In this current issue of Annals of Surgical Oncology, Zheng and colleagues report on a large institutional series of patients who underwent either minimally invasive or open McKeown esophagectomy. ${ }^{1}$ They analyze a variety of outcomes but mainly focus on long-term oncologic outcomes, specifically overall survival (OS) and disease-free survival (DFS). Not surprisingly, the authors report excellent overall outcomes and demonstrate yet again that MIE is, at minimum, equivalent to open esophagectomy (OE). In fact, the authors report that MIE led to improved OS compared with $\mathrm{OE}$ in their cohort of patients.

As early reports of MIE series were published, the procedure gained increasing acceptance. In a 2003 landmark report, Luketich et al. published the first large series of MIE. $^{2}$ This report of 222 patients showed that MIE can be performed with very low morbidity and mortality. In 2012, Briere et al. published the results of the Traditional Invasive vs. Minimally Invasive (TIME) trial, ${ }^{3}$ a multicenter, randomized controlled trial that showed clear shortterm benefits of MIE, including less postoperative pulmonary complications and shorter length of stay. In a follow-up publication, Straatman et al. reported the 3-year follow-up of the TIME trial and found no statistical difference in 3-year DFS and OS for MIE and OE. ${ }^{4}$ In fact, MIE was shown to have superior DFS and OS (although

(C) Society of Surgical Oncology 2021

First Received: 28 April 2021

Accepted: 1 May 2021;

Published Online: 23 June 2021

Z. Hammoud, MD

e-mail: zane.hammoud@ascension.org not enough to be statistically significant). In addition to the above reports, several meta-analyses have been published that have consistently demonstrated excellent outcomes for MIE compared with OE. ${ }^{5}$ Recently, a large series from a national database in Japan reported outcomes of 12,711 MIEs compared with 11,512 OEs. ${ }^{6}$ Short-term outcomes were excellent, leading the authors to state that "MIE can replace $\mathrm{OE}$ in various situations from the perspective of short-term outcomes".

No discussion on MIE is complete without a discussion regarding technique. Zheng et al. describe their MIE technique with thoracoscopic esophageal mobilization, laparoscopic creation of a gastric conduit, and left neck anastomosis, i.e. McKeown esophagectomy. However, it should be noted that this is not the most commonly performed MIE (at least in Western countries). The Ivor Lewis esophagectomy is performed more frequently for a variety of reasons, not the least of which is the fact that the majority of esophageal cancers seen in the Western hemisphere are distal adenocarcinomas. Nonetheless, a minimally invasive Ivor Lewis esophagectomy can be performed with equally excellent outcomes. Furthermore, while anastomotic techniques are beyond the scope of this commentary, several types of anastomotic techniques can be performed in an MIE (intrathoracic as well as left neck). Regardless of which type of esophagectomy is performed, published reports have shown consistently good outcomes with minimally invasive techniques.

The introduction of robotic assistance in MIE must also be mentioned. Robotic-assisted MIE (RAMIE) has also gained increasing acceptance. Potential advantages, benefits, and excellent short- and long-term outcomes have been reported with RAMIE.

In summary, MIE has obvious advantages compared with OE in terms of short-term outcomes, such as fewer pulmonary complications, shorter length of stay, and quicker recovery. In addition, excellent long-term functional and oncologic outcomes can be achieved with MIE 
that are at least equivalent to OE. Taken together, it is safe to conclude that MIE should be the esophagectomy operation of choice. All surgeons performing esophageal resection must adapt to this reality and work diligently to gain the skillset required to perform MIE.

\section{REFERENCES}

1. Zheng Y, Li Y, Liu X, et al. Minimally invasive versus open McKeown for patients with esophageal cancer: a retrospective study. Ann Surg Oncol. 2020;2:16.

2. Luketich JD, Alvelo-Rivera M, Buenaventura PO, et al. Minimally invasive esophagectomy: outcomes in 222 patients. Ann Surg. 2003;238(4):486-94.

3. Briere SS, van Borge Henegouwen MI, Maas KW, et al. Minimally invasive versus open oesophagectomy for patients with oesophageal cancer: a multi-centre, open-label, randomized controlled trial. Lancet. 2012;379:1887-92.

4. Straatman J, van der Wielen N, Cuesta M, et al. Minimally invasive versus open esophageal resection; three year follow up of the previously reported randomized controlled trial: the TIME trial. Ann Surg. 2017;266:232-6.

5. Guo W, Ma X, Yang S, et al. Combined thoracoscopic-laparoscopic esophagectomy versus open esophagectomy: a metaanalysis of outcomes. Surg Endosc. 2016;30:3873-81.

6. Yoshida N, Yamamoto $\mathrm{H}, \mathrm{Baba} \mathrm{H}$, et al. Can minimally invasive esophagectomy replace open esophagectomy for esophageal cancer? Latest analysis of 24,233 esophagectomies from the Japanese National Clinical Database. Ann Surg. 2020;272:118-24.

Publisher's Note Springer Nature remains neutral with regard to jurisdictional claims in published maps and institutional affiliations. 